\title{
Lion and Man Game in the Presence of a Circular Obstacle
}

\author{
Nikhil Karnad ${ }^{\dagger}$ and Volkan Isler
}

\begin{abstract}
In the lion and man game, a lion tries to capture a man who is as fast as the lion. We study a new version of this game which takes place in a Euclidean environment with a circular obstacle. We present a complete characterization of the game: for each player, we derive necessary and sufficient conditions for winning the game. Their (continuous time) strategies are constructed using techniques from differential games and arguments from geometry. Our main result is a decision algorithm which takes arbitrary initial positions as input, declares one of the players as the winner of the game and outputs a winning strategy for that player. We extend our approach to explicitly construct, in closed form, the decision boundary that partitions the arena into win and lose regions.
\end{abstract}

\section{OVERVIEW AND RELATED WORK}

In a game of pursuit and evasion, one player (the pursuer) tries to get close to, and possibly capture the other (the evader). The evader, in turn, tries to avoid being captured. Pursuit-evasion games are of fundamental importance to researchers in the field of robotics. Consider the task of surveillance, where a guard (pursuer) has to chase and capture an intruder (evader). Another scenario is search-andrescue, where a rescue worker has to locate a lost hiker. Since the actions of the hiker are not known a priori, worstcase pursuit and evasion strategies guarantee that the hiker is found no matter what he does. Problems arising from diverse applications such as collision-avoidance [9], search-andrescue [6], [14], air-traffic control [3], and surveillance [9] have been modeled as pursuit-evasion games.

A classical pursuit-evasion game is the Lion and Man game. It was originally posed in 1925 by Rado as follows

A lion and a man in a closed arena have equal maximum speeds. What tactics should the lion employ to be sure of his meal?

The first solution was generally accepted by 1950: the lion moves to the center of the arena and then remains on the radius that passes through the man's position. Since they have the same speed, the lion can remain on the radius and simultaneously move toward the man. Although this strategy works in discrete-time, it was later shown by Besicovitch that exact capture in continuous time takes infinitely long in a bounded arena [13]. However, if the capture distance is set to some $c>0$, Alonso et al. [2] showed that the lion can capture the man in time $O\left(\frac{r}{s} \log \frac{r}{c}\right)$, where $r$ is the radius

\footnotetext{
${ }^{\dagger}$ Nikhil Karnad is with the Department of Computer Science and Engineering at the University of Minnesota, 200 Union St SE, Minneapolis, Minnesota 55455, USA. karnadecs . umn. edu

Volkan Isler is with the Department of Computer Science and Engineering at the University of Minnesota, 200 Union St SE, Minneapolis, Minnesota 55455, USA. isler@cs . umn.edu

This work was supported by the grants NSF CCF-0634823 and NSF IIS-0745537.
}

of the circular arena and $s$ is the maximum speed of the players. In [15], Sgall studied the discrete time, continuous space variant in a semi-bounded environment: the positive quadrant. He showed that the lion captures the man, if certain invariants are satisfied initially.

Recently, researchers have studied variants of the lion and man game played in environments more complex than a circular disc or the real plane. Isler et al. showed that the lion can capture the man in any simply-connected polygon [10]. Alexander et al. presented a sufficient condition for the greedy strategy to succeed in arbitrary dimensions [1].

The lion and man game in the presence of obstacles remains a challenge. In this paper, we take an important step for solving the lion and man game in an environment with obstacles. We present full characterization of the game in the presence of a single circular obstacle. That is, we present a decision algorithm which determines the winner of the game. We also construct the winner's strategy.

As in the original version of the game, we assume that the players know exact locations of each other at all times and have equal maximum speeds.An important line of research is to study the effect of sensing limitations. Recent progress in this direction includes the study of range-based limitations [5] and bearing-based limitations [11].

Other variants of pursuit-evasion games studied in the robotics community are visibility based pursuit-evasion [8] and maintaining the visibility of an adversarial target [4].

\section{CONTRIBUTIONS}

We study the lion and man game played in a convex polygonal environment, where both time and space are continuous. There is a single circular obstacle in the environment. The main question we study is: Given initial locations of the players, which player wins the game?

In earlier work [10], researchers have shown that the pursuer can capture the evader in any simply-connected polygon. This intuitively suggests that the evader has to reach the obstacle to win the game. Conversely, the pursuer wins the game if he can separate the obstacle from the evader, and simultaneously make progress toward capture.

Verifying these conditions from an arbitrary initial configuration is difficult. For example, it is easy to see that the evader wins the game if he is closer to the obstacle. However, the condition is not necessary, as shown by the following instance. Consider a circular obstacle $O$ with center $A$ and radius 10 units. (see Fig. 1). The initial configuration is such that the pursuer $P$ and the evader $E$ are separated by a relative angle of $\pi$ radians, measured w.r.t. $A$. The closest point on the obstacle $O$ to the evader is $F$, such that $|E F|=10 \pi=|\overparen{P F}|$. 


\section{CONFIDENTIAL. Limited circulation. For review only.}

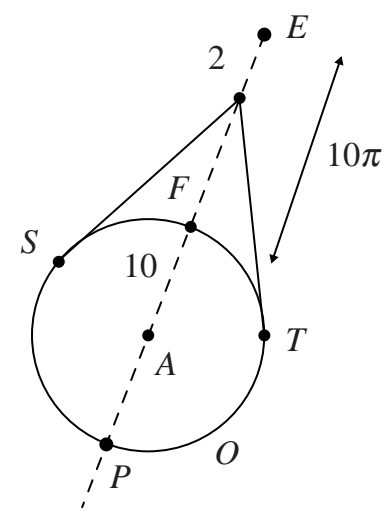

Fig. 1. A counterexample: The evader wins even though the pursuer is closer to all points on the obstacle.

Thus every point on the obstacle is closer to the pursuer than the evader.

If the evader heads straight to $F$ along $E F$, the pursuer picks one of the directions (clockwise or counterclockwise) to go around the obstacle and reaches $F$ at the same time as the evader, resulting in capture and a pursuer-win. This is true for any point $F \in \delta O$ if the evader just heads straight to $F$ from $E$ along the shortest path. However, consider the following strategy: the evader heads toward $F$ for 2 units and switches direction toward the tangent point that is farther from the pursuer. For instance, if the pursuer picks the clockwise direction to go around the obstacle, the evader heads to $T$. The time taken by the pursuer to reach $T$ in the clockwise direction is computed as 44.55 units, whereas the evader reaches $T$ at time 40.126 units. Thus the evader hits the obstacle at $T$ faster than the purser and then continues to loop around the obstacle away from the pursuer. Since the evader avoids capture indefinitely, the evader wins the game.

In this paper, we present a complete characterization to determine the outcome of the pursuit-evasion game for any given initial condition. Our results are organized as follows. In Section III, we formulate the task of the pursuer guarding the obstacle from the evader as a differential game. In Section IV-A, we use concepts from optimal control theory to derive optimal control laws. We provide the geometric interpretation of the player strategies in Section IV-B. In Section V, we derive necessary and sufficient conditions for each player to win the game. Our main result provides a decision algorithm for the pursuit-evasion problem, presented in Section VI. We extend the win-condition to derive an explicit expression for the decision boundary: in Section VII, we present a partitioning of the arena into a pursuer-win region and an evader-win region, for a given initial evader location. We conclude in Section VIII and suggest directions for future research.

\section{PROBlEM STATEMENT AND FORMULATION}

An evader $E$ and a pursuer $P$ are playing a game of pursuit and evasion inside a simply-connected convex polygon $\mathscr{P}$, with a single circular obstacle $O$. We say that the pursuer captures the evader if the geodesic distance between the

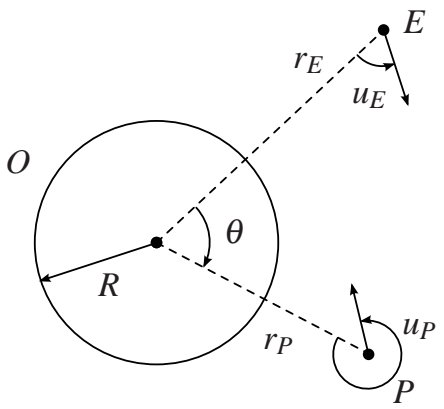

Fig. 2. The pursuer $P$ guarding the obstacle $O$ from the evader $E$.

players goes to zero as time goes to infinity. On the other hand, if the evader can guarantee a non-zero lower-bound on the distance between the players, the outcome of the game is an evader-win.

The game is played in continuous time and continuous space.

Let $R$ be the radius of $O$ (see Fig. 2). At any time $t \in[0, \infty[$, the state of the game is defined by three variables:

$$
\mathbf{x}(t)=\left[r_{P}(t), r_{E}(t), \theta(t)\right]^{T}
$$

where $\theta(t)$ is angle between the players $E$ and $P$, subtended at the center of $O$. The radial distance of $P$ from the center of $O$ is denoted as $r_{P}(t)$ and that of $E$ is $r_{E}(t)$. We drop the dependency on time from the notation and just use $r_{P}, r_{E}$ and $\theta$ where appropriate.

Both players are modeled as point objects with the same maximum speed, $v=1$. This is done by scaling all distances w.r.t. the value of $v$ (normalization). The pursuer $P$ (respectively the evader $E$ ) can pick a direction relative to his/her radius $r_{P}$ (respectively $r_{E}$ ) to move along. This is the control input $u_{P}(t)$ (resp. $u_{E}(t)$ ), where $u_{P}(t), u_{E}(t) \in S^{1} \forall t$. We study a game of complete information: both players know each others' locations at all times.

The kinematic equations (KE) for the state are

$$
\begin{aligned}
\dot{r}_{P} & =\cos u_{P} \\
\dot{r}_{E} & =\cos u_{E} \\
\dot{\theta} & =\frac{\sin u_{E}}{r_{E}}-\frac{\sin u_{P}}{r_{P}}
\end{aligned}
$$

The players occupy the part of the polygon outside of the obstacle $O$. Thus $r_{P}(t) \geq R, \forall t$ and $r_{E}(t) \geq R, \forall t$. We restrict the relative angle between the players as $\theta \in[-\pi, \pi]$

In order to win, the evader must guarantee a lower-bound on the distance between the players. This happens when the evader reaches the boundary of the obstacle $O$ without getting captured. In fact, this is the only way the evader can win the game as we will see in Section V-B. On the other hand, if the pursuer can prevent the evader from reaching $O$ and simultaneously make the distance between them go to zero, the pursuer will win the game. The pursuer can do so, if he is able to make the relative angle $\theta$ go to zero before the evader hits $O$ (This statement is formalized and proven as Theorem 1 of Section V-B.). We use these observations to formulate an equivalent game with the following objective. 


\section{CONFIDENTIAL. Limited circulation. For review only.}

Suppose the evader $E$ hits the boundary of $O$ at time $T \geq$ 0, i.e. $r_{E}(T)=R$. Then, the value of $\theta(T)$ describes the outcome of the game: if $|\theta(T)| \neq 0$, we know that $E$ reached $O$ before $P$ and thus $E$ wins the game. If not, we will show that the pursuer can align himself with the evader before $T$ and proceed to win the game by playing the Lion's strategy (see Theorem 1). The objective, or value, of the game is thus given by

$$
J=|\theta(T)|
$$

where

$$
T=\min \left\{t: r_{E}(t)=R\right\}
$$

We wish to solve the optimal control problem: what should be $u_{P}^{*}(t)$ and $u_{E}^{*}(t)$ so that $E$ maximizes $J$ and $P$ minimizes it? It is worth noting that we study the game of kind, and seek strategies that are optimal in terms of the outcome of the game.

This problem falls in the context of differential games. Although the solution process is along the lines of the Lady in the Lake problem (see [3], Sec. 8.5, pp. 452-456), our problem is significantly different: if both the lady and the man had equal velocities, the lady would always win the game by swimming along the line joining them, in the direction away from the man. In contrast, the outcome of our game, depends on the initial conditions.

\section{OPTIMAL PLAYER STRATEGIES}

In this section, we use optimal control theory, in the realm of differential games, to derive the optimal strategies for the pursuer and evader. Further, we present the geometric interpretation of the strategies.

\section{A. Optimal control solution}

Let $\mathbf{x}(t)$ be the state vector, and $\mathbf{u}(t)$ the control input. Optimizing an objective function of the form

$$
J(u)=h(\mathbf{x}(T), T)+\int_{t_{0}}^{t_{f}} g(\mathbf{x}(t), \mathbf{u}(t), t) d t
$$

with a terminal payoff $h(\cdot)$ and an integral payoff $g(\cdot)$ subject to the KE (1) is equivalent (Pontryagin's Maximum Principle) to optimizing the Hamiltonian $H$ given by

$$
H(\mathbf{x}(t), \mathbf{u}(t), \mathbf{p}(t), t)=g(\mathbf{x}(t), \mathbf{u}(t), t)+\mathbf{p}^{T}(t)[\mathbf{a}(\mathbf{x}(t), \mathbf{u}(t), t)]
$$

where $\mathbf{p}(t)$ is a vector of Lagrange multipliers, also known as the costate variables. $\mathbf{a}(t)$ is the vector of state transition equations from the $\mathrm{KE}(1)$.

In our problem, we only have a terminal payoff $h(\mathbf{x}(T), T)=|\theta(T)|$ and no integral payoff. Thus the Hamiltonian for our system is

$$
\begin{aligned}
H & =p_{P}(t) \dot{r}_{P}(t)+p_{E}(t) \dot{r}_{E}(t)+p_{\theta}(t) \dot{\theta}(t) \\
& =p_{P}(t) \cos u_{P}(t)+p_{E}(t) \cos u_{E}(t) \\
& +p_{\theta}(t)\left(\frac{\sin u_{E}(t)}{r_{E}(t)}-\frac{\sin u_{P}(t)}{r_{P}(t)}\right)
\end{aligned}
$$

The Isaacs equation is

$$
\min _{u_{P}} \max _{u_{E}} H=0
$$

We use a standard result from optimal control theory to obtain necessary conditions for $u_{P}^{*}$ and $u_{E}^{*}$ to optimize the Hamiltonian (see [12], 5.1-17b, pp. 187-188). For all time $t$ the following is true.

$$
\begin{aligned}
\dot{\mathbf{x}}^{*}(t) & =\frac{\partial H}{\partial \mathbf{p}} \\
\dot{\mathbf{p}}^{*}(t) & =-\frac{\partial H}{\partial \mathbf{x}} \\
\mathbf{0} & =\frac{\partial H}{\partial \mathbf{u}}
\end{aligned}
$$

Since the final state at time $T$ is free, except for $r_{E}(T)=R$, we have additional boundary conditions (commonly referred to as transversality conditions) given by

$$
\frac{\partial h}{\partial \mathbf{x}}\left(\mathbf{x}^{*}(T), T\right)-\mathbf{p}^{*}(T)=\mathbf{0}
$$

Applying these necessary conditions to our problem, we have

$$
\begin{aligned}
\dot{p}_{P}^{*}(t) & =-\frac{\delta H}{\delta r_{P}}=-\frac{p_{\theta}^{*} \sin u_{P}^{*}}{r_{P}^{* 2}} \\
\dot{p}_{E}^{*}(t) & =-\frac{\delta H}{\delta r_{E}}=\frac{p_{\theta}^{*} \sin u_{E}^{*}}{r_{E}^{* 2}} \\
\dot{p}_{\theta}^{*}(t) & =-\frac{\delta H}{\delta \theta}=0 \\
0 & =\frac{\delta H}{\delta u_{P}}=-p_{P}^{*} \sin u_{P}^{*}-\frac{p_{\theta}^{*}}{r_{P}^{*}} \cos u_{P}^{*} \\
0 & =\frac{\delta H}{\delta u_{E}}=-p_{E}^{*} \sin u_{E}^{*}+\frac{p_{\theta}^{*}}{r_{E}^{*}} \cos u_{E}^{*}
\end{aligned}
$$

The boundary (transversality) condition is

$$
\begin{aligned}
\frac{\partial h(T)}{\partial \theta}-p_{\theta}^{*}(T) & =0 \\
\Rightarrow p_{\theta}^{*}(T) & =\frac{\partial(|\theta(T)|)}{\partial \theta}=\operatorname{sgn}(\theta(T))
\end{aligned}
$$

From (3) and (4) we have $\forall t, p_{\theta}^{*}(t)=\operatorname{sgn}(\theta(T))$. Solutions for $u_{P}^{*}$ and $u_{E}^{*}$ that optimize $H$ are

$$
\begin{aligned}
& \left(\sin u_{P}^{*}, \cos u_{P}^{*}\right) \|\left(-\frac{p_{\theta}^{*}}{r_{P}^{*}}, p_{P}^{*}\right) \\
& \left(\sin u_{E}^{*}, \cos u_{E}^{*}\right) \|\left(\frac{p_{\theta}^{*}}{r_{E}^{*}}, p_{E}^{*}\right)
\end{aligned}
$$

Let the constants of proportionality for the parallel vectors be $c_{P}$ and $c_{E}$. Substitute in the Isaacs equation (2) to get $c_{P}=-c_{E}$. Now use the evader's boundary condition in (5):

$$
c_{E} \sin u_{E}^{*}=\frac{p_{\theta}^{*}}{r_{E}^{*}}
$$

At $t=T$, the evader hits the boundary of the obstacle i.e. $r_{E}(T)=R$. The evader wins the game then on by remaining on the boundary of $O$ and moving in the direction that takes him away from the pursuer i.e. his velocity vector is tangent to $O$ thereafter. In terms of his control variable $u_{E}$, we have $u_{E}(T)=\operatorname{sgn}(\theta(T)) \cdot \frac{\pi}{2}$. Therefore

$$
\begin{aligned}
c_{E} \operatorname{sgn}(\theta(T)) & =\frac{\operatorname{sgn}(\theta(T))}{R} \\
\Rightarrow c_{E} & =R^{-1}
\end{aligned}
$$




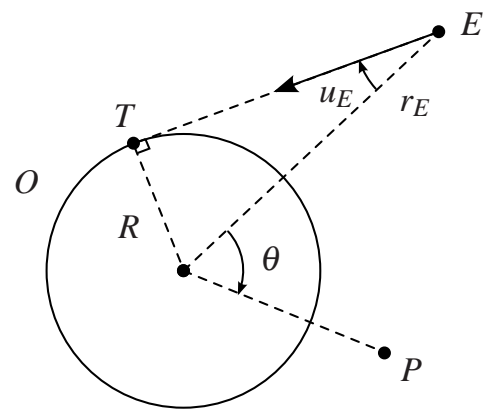

Fig. 3. $E$ moves away from $P$ along a tangent to $O$.

Finally, solve for $u_{P}^{*}$ and $u_{E}^{*}$ by substituting known values into (5).

$$
\begin{aligned}
r_{P}(t) \sin u_{P}^{*}(t) & =-r_{E}(t) \sin u_{E}^{*}(t) \\
& =R \operatorname{sgn}(\theta(T))
\end{aligned}
$$

\section{B. Geometric interpretation}

To understand the physical meaning of (6), consider the evader: $r_{E}(t), u_{E}(t)$. The argument holds for the pursuer using $r_{P}(t), u_{P}(t)$ instead.

$$
\sin u_{E}^{*}(t)=-\frac{R}{r_{E}(t)} \operatorname{sgn}(\theta(T))
$$

Let $Q$ be the center of the obstacle $O$ (see Fig. 3). Consider triangle $\triangle Q E T$, where $T$ is the point on $O$ where the tangent from $E$ touches it. Clearly, Equation (6) is satisfied, meaning that the solution for the evader $E$ is to always head toward $O$ along the direction of the tangent from $E$ to $O$. Since there are two possible tangents for any evader location, he picks the one in accordance with the value of $\operatorname{sgn}(\theta(T))$. Since $\theta \in[-\pi, \pi]$, we know that he will pick the tangent in such a way that his direction of motion makes the value of the relative angle $\theta$ greater.

The pursuer $P$, being the minimizing player, moves along the tangent circle in the direction that reduces the relative angle between the players.

\section{DECIDING A WINNER}

Given an initial condition for our game, and the optimal strategies derived in Section IV, we characterize under which conditions the game ends in a pursuer-win and an evader-win.

First, we use a direct observation to eliminate one case: when the evader is initially closer to the obstacle than the pursuer. If $r_{E}(0)<r_{P}(0)$, the evader $E$ reaches the boundary of the obstacle before the pursuer $P$ simply by heading directly to the point on the obstacle closest to $E$. Thereafter, the evader loops around the obstacle and avoids being captured indefinitely, leading to an evader-win.

Next, consider the case when $r_{E}(0) \geq r_{P}(0)$. Let the initial relative angle be $\theta(0)=\theta_{0}$, say. If $\theta_{0}= \pm \pi$, the pursuer can

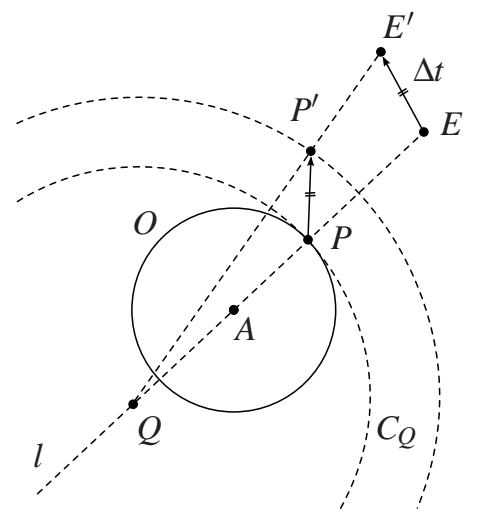

Fig. 4. An illustration of the Lion's move used by the pursuer $P$ to stay on the evader's radial line w.r.t. $Q$.

pick either direction to go around $O$. If not, he picks the smaller of the angles. Once he picks a direction, the pursuer will not switch. This is because by switching directions, the pursuer allows the relative angle $\theta$ to increase while the evader is decreasing his distance to the obstacle. The evader would then be in a better configuration i.e. same angular separation as before, but with a lesser distance to the the obstacle.

\section{A. Time to termination}

The time at which the evader hits the obstacle $\left(T_{h i t}\right)$ is the length of the tangent w.r.t. his initial location, since he moves with unit velocity. By Pythagoras' theorem, we get

$$
T_{h i t}=\sqrt{r_{E}(0)^{2}-R^{2}}
$$

The pursuer wins the game if he can align himself with the evader. The time at which this happens is $T_{\text {align }}$ such that $\theta\left(T_{\text {align }}\right)=0$. If $T_{\text {hit }}<T_{\text {align }}$, the evader reaches the obstacle before the pursuer prevents him, and thus the evader wins the game. If not, the pursuer can align himself with the evader, after which the pursuer can execute the Lion's strategy and win the game. The Lion's strategy guarantees capture for the pursuer when he stays on the radius of a growing circle, between a fixed center point and the evader's location (see Fig. 4). A description of this pursuit strategy in continuous time can be found in [13].

There are two ways to compute $T_{\text {align }}$. First, we can integrate the relative angle between the players (Equation (1)) from $\theta(0)$ to 0 as time goes from 0 to $T_{\text {align. }}$. This method is presented in Appendix A. The second method, uses arguments from geometry and worst-case player strategy analysis, comparing the total lengths of the paths that each player takes. We present the second method as follows.

Given the optimal strategies for both players, we know the exact points of tangency of their trajectories w.r.t. the obstacle. This allows us to compute the time taken by the pursuer $P$ to reach the point of tangency of the evader $E$. Let us call that point as $F$.

If the pursuer does not align himself with the evader by this time, the evader wins because he hits the obstacle without getting captured. We therefore have an upper bound 


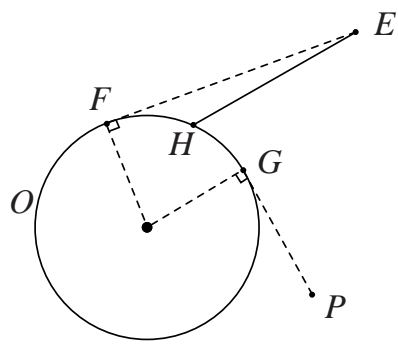

(a)

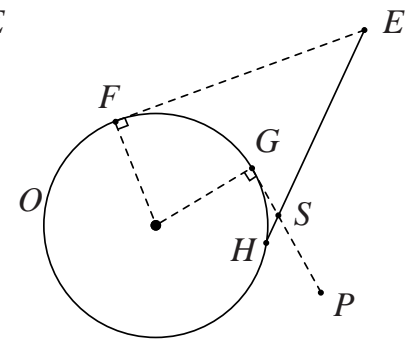

(b)
Fig. 5. Different cases for when the evader hits the obstacle at $H$ : (a) $H$ is on $\overparen{G F}$, and, (b) $E H$ intersects $P G$ at $S$.

on the alignment time $T_{\text {align }}$. The time taken by a pursuer traveling at unit velocity to reach the point of tangency $F$ of the evader w.r.t. the obstacle $O$ is the sum of the length of the pursuer's tangent to $O$, and, the length of the arc from the pursuer's point of tangency to $F$ (see Fig. 5).

For the rest of the paper, instead of denoting the time at which $\theta(t)$ becomes 0 as $T_{\text {align }}$, we let $T_{\text {align }}$ be the time at which the pursuer reaches the point $F$. In essence, if $P$ reaches $F$ before $E$ reaches $F$, we have that the pursuer wins. However, the exact time of alignment lies in the interval $\left[0, T_{\text {align }}\right]$, provided the evader plays his optimal strategy. In the following section, we show that no matter what the evader does, the game is decided just by comparing the time both players take to reach the point $F$. Our denotation of $T_{\text {align }}$ as the time taken to reach $F$ is therefore justified.

\section{B. Pursuer-win condition}

In general, the evader may choose not to play the optimal strategy, which affects two aspects of the game: (i) the point on the boundary of the obstacle $O$ at which the evader hits $O$, and, (ii) the time at which an alignment possibly occurs. In this section, we show that whenever $T_{\text {align }} \leq T_{h i t}$, the pursuer wins the game irrespective of what the evader does with (i) and (ii).

For the rest of our discussion, we adopt the following notation (see Figure 5). Let $P$ be the pursuer's initial location and $E$ that of the evader. Let $F$ be the point of tangency of $E$ w.r.t. $O$ in the direction he heads away from $P$ to increase the relative angle between the players. Let $G$ be the point of tangency, w.r.t. $O$, of the pursuer along the direction he heads to decrease the relative angle between the players.

As derived earlier, $T_{h i t}$ is the length of line segment $E F$. Further, $T_{\text {align }}$ is given by $P G+\overparen{G F}$.

Lemma 1: The following two statements $S_{1}$ and $S_{2}$ are equivalent.

$S_{1}: T_{\text {align }} \leq T_{\text {hit }}$

$S_{2}$ : The pursuer can align with the evader in finite time i.e. the relative angle between the players goes to zero in finite time, irrespective of the evader's actual trajectory. Proof: $S_{2} \Rightarrow S_{1}$ : Let the relative angle between the players go to zero in finite time. The evader cannot be on the boundary of the obstacle unless the pursuer is coincident with him. If not, the evader can always maintain angular separation from the pursuer by looping around the obstacle along the boundary of $O$, maintaining a non-zero lower-bound on the relative angle between the players - a contradiction. Thus the pursuer aligns himself with the evader before, or exactly at, the time the evader hits the obstacle. When the evader hits the obstacle at the point $F$, we have $T_{\text {align }} \leq T_{\text {hit }}$ by definition. $S_{1} \Rightarrow S_{2}$ : Suppose $T_{\text {align }} \leq T_{\text {hit }}$. By definition, we have

$$
P G+\overparen{G F} \leq E F
$$

We have two possible cases: either the evader hits the obstacle at some finite time, or, he does not hit the obstacle ever. In the second case, the pursuer has a higher angular velocity than the evader about the center $O$ and since the evader does not hit the obstacle, the relative angle between them goes to zero (players are aligned) in finite time.

We now focus on the first case: the evader hits the obstacle at some point, call it $H$. We show that the pursuer is closer to $H$ no matter where $H$ lies on the boundary of $O$. Thus the pursuer can reach $H$ before the evader no matter where $H$ lies on $O$. Note that we consider that the evader takes the shortest possible path to reach $H$, but our argument holds for any evader strategy that takes him to $H$, because it will take him at least as long as the time along the shortest path to $H$.

Case 1. $H$ lies on $\overparen{G F}$ (see Fig. 5 (a)). Expand (8)

$$
\begin{aligned}
& P G+\overparen{G F} \leq E F \\
\Rightarrow & P G+\overparen{G H}+\overparen{H F} \leq E F \\
\Rightarrow & P G+\overparen{G H} \leq E F-\overparen{H F}
\end{aligned}
$$

Use triangle inequality in $\triangle E F H$, where $F H$ is a chord of the obstacle $O$ i.e. $F H<\overparen{F H}$ to get

$$
\begin{array}{r}
P G+\overparen{G H} \leq E F-H F \\
\Rightarrow P G+\overparen{G H} \leq E H
\end{array}
$$

Thus the pursuer is closer to $H$ than $E$ and thus can align himself with the evader before the evader reaches $H$.

Case 2. $H$ lies on the boundary of $O$ such that $E H$ intersects $P G$. Call the point of intersection as $S$ (see Fig. 5 (b)). Expand (8)

$$
\begin{array}{r}
P G+\overparen{G F} \leq E F \\
\Rightarrow P S+S G+\overparen{G F} \leq E F \\
\Rightarrow P S \leq E F-S G-\overparen{G F}
\end{array}
$$

Use triangle inequality in $\triangle S G F$, where $S$ lies outside the circle $O$ such that $F S$ is a secant of $O$ i.e. $S F<S G+\overparen{G F}$ to get

$$
P S \leq E F-S F
$$

Finally, triangle inequality in $\triangle E F S$ gives us

$$
P S \leq E S
$$




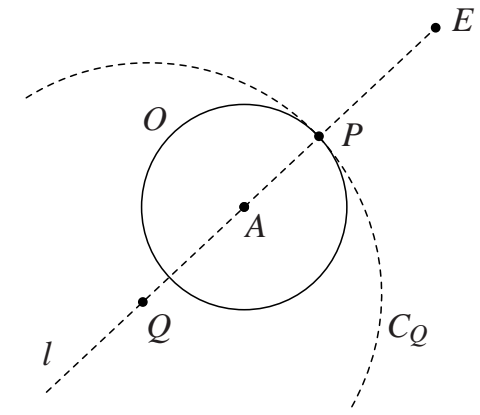

Fig. 6. Existence of a separating circle when the players are aligned.

Thus the pursuer is closer to $S$ than $E$ and thus can align himself with the evader before the evader reaches $S$ (and thereby $H$ ).

Case 3. $H$ lies on the part of the boundary of $O$ beyond $F$. The shortest path from $E$ to $H$ wraps around $F$ : it is $E F \cdot \overparen{F H}$. The shortest path from $P$ to $H$ wraps around $G$ and is given by $P G \cdot \overparen{G F} \cdot \overparen{F H}$. Since $P G+\overparen{G F} \leq E F$, adding $\overparen{F H}$ gives us the required result: $P$ is closer to $H$ than $E$ and thus reaches $H$ before $E$. Since $E$ hits $O$ at $H$, we have that the players are aligned.

In all of the cases, we observe that the pursuer is closer to all points on the obstacle than the evader when the condition $T_{\text {align }} \leq T_{\text {hit }}$ is true. Consequently, he can align himself with the evader in finite time.

Lemma 1 results in a configuration of the game where the pursuer and evader are radially aligned w.r.t. $O$ such that the pursuer is closer to the center of $O$ than the evader. We show that from this point on, the pursuer wins the game by following the Lion's strategy, adapted to a simply-connected polygon (as explained in [10]). We summarize this result in the following lemma by proving that the initial conditions for the existence of a winning pursuer strategy are satisfied at the time of alignment.

Lemma 2: When the players are aligned radially w.r.t. $O$, with the pursuer closer to $O$ than the evader, the pursuer wins the game by following the Lion's strategy.

Proof: First, we show that there exists a circle that separates the pursuer from the evader, constructed as follows (see Fig. 6).

Suppose the pursuer is at $P$ and the evader at $E$. Let the center of the obstacle $O$ be $A$. Let $l$ be the line passing through $E, P$ and $A$. Pick a point $Q$ on $l$ such that a circle $C_{Q}$ centered at $Q$ passes through $P$ and completely contains $O$. For example, if $Q$ coincides with $P$, then $O$ is the same as $C_{Q}$. We can pick any other point $Q$ on $l$ farther away from $P$ than $A$ and that will work as well. The other extreme is when $Q$ is at infinity on $l$, at which point $C_{Q}$ degenerates to the tangent to $O$ at $P$. Therefore, such a circle always exists when the players are aligned.

The pursuer follows the Lion's strategy, depicted in Fig. 4: he always remains on the radius $C E^{\prime}$ for an evader move from $E$ to $E^{\prime}$ (say). This sandwiches the evader between the boundary of the polygonal arena $\mathscr{P}$ and a growing circle $C_{Q}$ with a fixed center $Q$. The continuous time Lion's strategy is discussed in [13]. For a detailed analysis of the discrete time version of the Lion's strategy in simply-connected polygons, the interested reader is directed to [10].

The game terminates at the boundary of the polygonal arena where the evader is captured. If the evader plays suboptimally, the game could terminate before he reaches the boundary as well, but the outcome of the game remains unchanged i.e. the pursuer wins by capturing the evader.

We combine Lemma 1 and Lemma 2 to state our main result.

Theorem 1: When $T_{\text {align }} \leq T_{\text {hit }}$, the pursuer wins the game by first aligning himself with the evader, then executing the Lion's strategy. If not i.e. if $T_{h i t}<T_{\text {align }}$, the evader reaches the obstacle and wins the game thereafter by looping around its boundary and avoiding capture indefinitely.

\section{DECISION ALGORITHM}

Let the initial configuration of the game be $G(0)=$ $\left(r_{P}(0), r_{E}(0), \theta(0), \mathscr{P}, O\right)$, where $r_{P}(0)$ is the initial radial distance of the pursuer from the center of the obstacle $O$ and $r_{E}(0)$ that of the evader. $\theta(0)$ is the initial relative angle between the players. $\mathscr{P}$ is a description of the simplyconnected polygonal arena that contains the obstacle $O$ and the players in its interior.

The radius of the circular obstacle $O$ is $R$, a given constant. Let the center of $O$, denoted by $A$, be the origin of our coordinate frame. We further set the positive $\mathrm{X}$-axis along the evader's radius, which makes the relative angle $\theta(t)$ the angle subtended by the pursuer's radius w.r.t. evader's radius for all time $t$.

We assume that a feasible description is provided i.e. unexpected conditions, such as the players starting from inside the obstacle, are not checked for. Our decision algorithm is listed as Algorithm 1. The three subroutines are used in our algorithm are

- Cartesian $(r, \theta)$

Converts from Polar coordinates to Cartesian coordinates.

- TangentOfEvader $(E, P)$

Computes the point on intersection of the tangent from the evader's location $E$ to the circular obstacle $O$, taking into account the value of $-\operatorname{sgn}(\theta)$ to decide which of the two possible tangents to use.

- TangentOfPursuer $(P, E)$

Computes the point on intersection of the tangent from the pursuer's location $P$ to the circular obstacle $O$, taking into account the value of $\operatorname{sgn}(\theta)$ to decide which of the two possible tangents to use.

\section{DECISION BOUNDARY}

The winning condition derived in Section $\mathrm{V}$ is a comparison of the length of the evader's tangent to the length of the pursuer's path to the evader's point of tangency. We can use this result to answer a more general question: given the evader's initial location $E$, a description of the polygon $\mathscr{P}$, and the obstacle $O$, what is the pursuer-win region? In other 


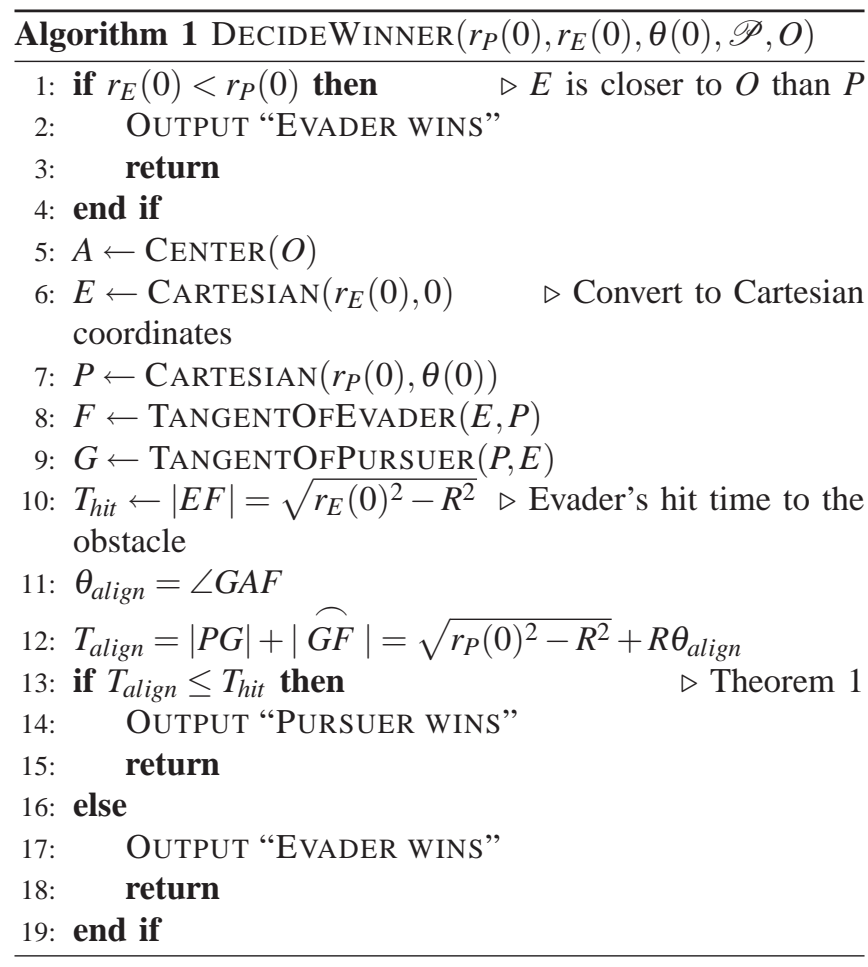

words, what is the boundary of the region within which the pursuer starts and wins the game, and outside of which the pursuer is unable to capture the evader?

Given an initial evader location $E$, the points of tangency from $E$ to $O$, call them $T$ and $S$ as before, are fixed and can be computed directly (see Fig. 7). Since the length of the evader's tangent is known, call it $w\left(w=T_{h i t}\right)$, we can compute the set of all points that are at most a distance of $w$ from $T$ and $S$. However, we need to account for the direction of traversal of the pursuer. For instance, we retain the part of the region of distance at most $w$ from $S$ that lies on the other side of the line $E O$ when compared to $S$ i.e. if the pursuer were to head toward $S$ from close by, the evader would rather head to $T$ and thus it is necessary to check the distance of the pursuer from $T$ rather than $S$. The region is symmetric about the line $E O$ (the evader's radius) because the evader can switch tangents when the relative angle between the players hits $\pi$.

The equation for the boundary of the region in polar form is $(r, \theta)$ where

$$
\begin{gathered}
r(\theta)=\left\{R^{2}+(w-R(\theta-\alpha))\right\}^{\frac{1}{2}} \\
\text { where, } \cos \alpha=\frac{R}{r}
\end{gathered}
$$

Fig. 7 was obtained by varying $\theta$ in discrete steps and computing the corresponding $r(\theta)$. The resulting region was plotted in Java and the snapshot produced.

It can be seen that the polar coordinate equation is of the form

$$
a \theta+b r^{2}+c \cos ^{-1} \frac{R}{r}+d=0
$$

where $a, b, c$, and $d$ are known constants. By substituting the initial radius and angle of the pursuer into the equation, we

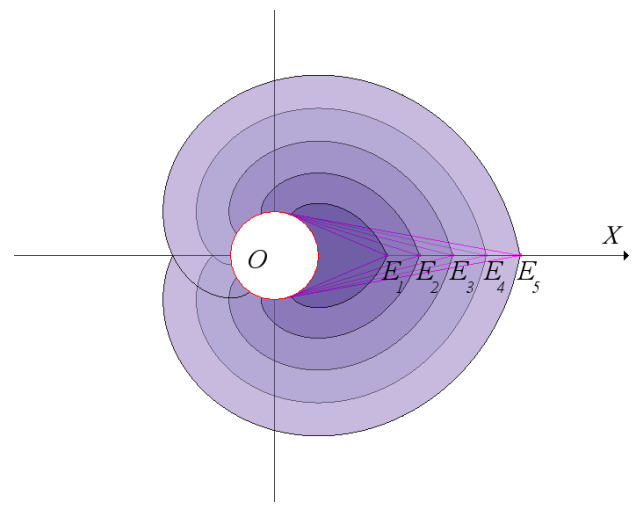

Fig. 7. For each evader location $E_{i}$ (moved along positive $X$ away from $O)$, the shaded regions are pursuer-win regions. The evader's tangents to $O$ are also shown.

can check on which side of the boundary the pursuer lies. In that sense, we call this equation the decision boundary. This gives us an alternate method for deciding which player wins the game.

In comparison, our solution approach in Section V uses lengths of simple curves (tangent and arc of a circle), and an analysis of worst-case strategies to derive a simpler decision formula.

\section{CONCLUSIONS AND FUTURE WORK}

We presented a decision algorithm for a pursuit-evasion game played in a convex polygonal arena with a circular obstacle: given a description of the environment, and the initial location of a pursuer and an evader, our algorithm determines which player wins the game. We extended the necessary and sufficient condition for winning the game to compute a partition of the arena into a pursuer-win region and an evader-win region. To the best of our knowledge, this is the first work in which a pursuit-evasion game has been completely characterized in the presence of a non-trivial obstacle.

Although both of our solution approaches (Section V and Section VII) are equivalent, the best solution depends on the application. For example, if we have control over where to deploy a guard (pursuer) to prevent an intruder (evader) from reaching his goal (obstacle), we might compute the decision boundary and pick the most suitable location from the interior of the pursuer-win region depending on other criteria. However, if the initial conditions have already been decided, we can just use the condition from Algorithm 1 to check whether capture is possible or not.

Researchers in the robotics community are interested in the incorporation of sensing limitations in pursuit-evasion games. For instance, visibility [8], [4], field-of-view [7], and range [5] limitations have been studied in contrast to complete information. These are interesting directions to explore. As part of immediate future work, we plan to extend our result to non-convex environments with multiple circular obstacles and polygonal obstacles. 


\section{CONFIDENTIAL. Limited circulation. For review only.}

\section{APPENDIX}

\section{A. Computing $T_{\text {align }}$ by integrating $\theta(t)$}

To compute the relative angle between the players $(\theta)$ as an explicit function of time, we first derive the evader's radial distance function $r_{E}(t)$.

The pursuer's trajectory follows the same geometric idea, differing only in the direction he picks to traverse around the obstacle. For simplicity of notation, let $r(t)=r_{E}(t) \forall t$ be the evader's radial distance from the center of $O$. Assume that $\operatorname{sgn}(\theta(T))=+1$ w.l.o.g. From (1) and (6), we have

$$
\dot{r}=\cos u_{E}^{*}=\sqrt{1-\sin ^{2} u_{E}^{*}}=\frac{\sqrt{r^{2}-R^{2}}}{r}
$$

Change the variable to $w$, where $r=R \csc w$ and integrate.

$$
r^{2}(t)=t^{2}+2 c_{1} R t+R^{2}\left(1+c_{1}^{2}\right)
$$

To solve for $c_{1}$, use the given initial value $r(0)=r_{E}(0)$.

$$
r(t)=\left(r_{E}(0)^{2}+t^{2}-2 t\left(\sqrt{r_{E}(0)^{2}-R^{2}}\right)\right)^{\frac{1}{2}}
$$

By definition, $T_{\text {align }}$ is the time at which the relative angle between the players reaches zero. From (1) and (6) we express the rate of change of the relative angle between the players as a function of their radial distances from the origin. The closed form solution for the latter was obtained as (9). The signs are chosen according to the direction of travel of the players.

$$
\dot{\theta}=-\frac{R \operatorname{sgn}(\theta(T))}{r_{E}^{2}}-\frac{R \operatorname{sgn}(\theta(T))}{r_{P}^{2}}
$$

Substitute for $r_{P}(t)$ and $r_{E}(t)$ from (9), separate the variables and integrate using the standard form

$$
\int \frac{d x}{x^{2}+a^{2}}=\frac{1}{a} \tan ^{-1} \frac{x}{a}
$$

We integrate from 0 to $T_{\text {align }}$, during which time the relative angle $\theta$ goes from $\theta(0)=\theta_{0}$ (say) to $\theta\left(T_{\text {align }}\right)=$ 0 . To reduce clutter, let $k_{1}=\sqrt{r_{P}(0)^{2}-R^{2}}$ and $k_{2}=$ $\sqrt{r_{E}(0)^{2}-R^{2}}$

$$
\operatorname{sgn}(\theta(T)) \cdot \theta_{0}=\tan ^{-1}\left[\frac{t-k_{2}}{R}\right]_{0}^{T_{\text {align }}}+\tan ^{-1}\left[\frac{t-k_{1}}{R}\right]_{0}^{T_{\text {align }}}
$$

Substitute the endpoints for $t$, let $k_{3}=\tan \left(\operatorname{sgn}(\theta(T)) \theta_{0}\right)$ and use the addition formula for inverse tangent ${ }^{1}$ :

$$
\tan ^{-1} u-\tan ^{-1} v=\tan ^{-1} \frac{u-v}{1+u v}
$$

to obtain the following quadratic in $T_{\text {align }}$

$$
\begin{array}{r}
T_{\text {align }}^{2}\left[k_{1} k_{2} k_{3}-k_{3} R^{2}+k_{1} R+k_{2} R\right] \\
+T_{\text {align }\left[-k_{1} k_{3} r_{E}(0)^{2}-k_{2} k_{3} r_{P}(0)^{2}-R r_{E}(0)^{2}-R r_{P}(0)^{2}\right]} \\
+k_{3} r_{P}(0)^{2} r_{E}(0)^{2}=0
\end{array}
$$

\footnotetext{
${ }^{1}$ To use this formula, we further need to verify that $|u v|<1$
}

Case 1. Consider the case when $\theta_{0}=0$ or $\theta_{0}= \pm \pi$ i.e. $k_{3}=0$. The quadratic simplifies to

$$
T_{\text {align }}^{2}\left[k_{2} R+k_{1} R\right]+T_{\text {align }}\left[-\operatorname{Rr}_{E}(0)^{2}-\operatorname{Rr}_{P}(0)^{2}\right]=0
$$

Giving us $T_{\text {align }}=0$, which corresponds to the case when $\theta_{0}=0$ i.e. the players are aligned to begin with. In the other case, divide by $T_{\text {align }} \neq 0$

$$
T_{\text {align }}=\frac{r_{P}(0)^{2}+r_{E}(0)^{2}}{k_{1}+k_{2}}
$$

Case 2. $\theta_{0}= \pm \frac{\pi}{2}$, in which case $k_{3}= \pm \infty$. Since $k_{3} \neq 0$, divide throughout by $k_{3}$ to get a new quadratic in which we let $k_{3} \longrightarrow \infty$ i.e. $\frac{1}{k_{3}} \longrightarrow 0$ to obtain

$$
\begin{aligned}
T_{\text {align }}^{2}\left[k_{1} k_{2}-R^{2}\right]+T_{\text {align }}\left[-k_{1} r_{E}(0)^{2}-\right. & \left.k_{2} r_{P}(0)^{2}\right] \\
& +r_{P}(0)^{2} r_{E}(0)^{2}=0
\end{aligned}
$$

which is a simplified quadratic in $T_{\text {align }}$.

In all other cases, the quadratic given by (11) applies. Thus the solution to (11) and the special cases listed above together allow us to solve for $T_{\text {align }}$ in closed form and compare the value to $T_{h i t}$ to decide which player wins the game. The equations are quadratics because the players can pick either direction to travel about the obstacle. The appropriate solution can be picked by ensuring that the pursuer moves along the lesser of the two relative angles between the players.

\section{REFERENCES}

[1] S. Alexander, R. Bishop, and R. Ghrist. Pursuit and evasion in nonconvex domains of arbitrary dimensions. In G. S. Sukhatme, S. Schaal, W. Burgard, and D. Fox, editors, Robotics: Science and Systems. The MIT Press, 2006

[2] L. Alonso, A. S. Goldstein, and E. M. Reingold. "Lion and man": upper and lower bounds. ORSA J. Comput., 4(4):447-452, 1992.

[3] T. Basar and G. J. Olsder. Dynamic Noncooperative Game Theory. Academic Press, London, revised 2nd edition, January 1995.

[4] S. Bhattacharya and S. Hutchinson. On the existence of nash equilibrium for a visibility based pursuit evasion game. In Workshop on Algorithmic Foundations of Robotics, 2008.

[5] S. D. Bopardikar, F. Bullo, and J. P. Hespanha. Sensing limitations in the Lion and Man problem. In ACC, pages 5958-5963, New York, July 2007.

[6] J. H. Discenza and L. D. Stone. Optimal survivor search with multiple states. Operations Research, 29(2):309-323, Mar.-Apr. 1981.

[7] B. P. Gerkey, S. Thrun, and G. Gordon. Visibility-based Pursuitevasion with Limited Field of View. The International Journal of Robotics Research, 25(4):299-315, 2006.

[8] L. J. Guibas, J. claude Latombe, S. M. Lavalle, D. Lin, and R. Motwani. Visibility-based pursuit-evasion in a polygonal environment. In International Journal of Computational Geometry and Applications, pages 17-30. Springer-Verlag, 1997.

[9] R. Isaacs. Differential Games. Wiley, New York, NY, 1965.

[10] V. Isler, S. Kannan, and S. Khanna. Randomized pursuit-evasion in a polygonal environment. IEEE Transactions on Robotics, 5(21):864$875,2005$.

[11] N. Karnad and V. Isler. Bearing-Only Pursuit. In Proc. IEEE Int. Conf. on Robotics and Automation, pages 2665-2670, May 2008.

[12] D. E. Kirk. Optimal control theory; an introduction. Prentice-Hall, Englewood Cliffs, N.J., 1970.

[13] J. E. Littlewood. A Mathematician's Miscellany. Methuen \& Co. Ltd. London, 1953.

[14] M. Prandini, J. P. Hespanha, and G. J. Pappas. Greedy control for hybrid pursuit games. In ECC01, pages 2621-2626, September 2001.

[15] J. Sgall. Solution of david gale's lion and man problem. Theor. Comput. Sci., 259(1-2):663-670, 2001. 